\title{
Archéopages
}

Archéopages

Archéologie et société

Hors-série 1 | 2008

Construction $^{s}$ de l'archéologie

\section{Évolution et professionnalisation de l'archéologie préventive en milieu rural dans le nord de la France}

Marc Talon

\section{(2) OpenEdition}

Journals

Édition électronique

URL : https://journals.openedition.org/archeopages/854

DOI : $10.4000 /$ archeopages.854

ISSN : 2269-9872

Éditeur

INRAP - Institut national de recherches archéologiques préventives

Édition imprimée

Date de publication : 1 février 2008

Pagination : 65-69

ISSN : $1622-8545$

\section{Référence électronique}

Marc Talon, «Évolution et professionnalisation de l'archéologie préventive en milieu rural dans le nord de la France », Archéopages [En ligne], Hors-série 1 | 2008, mis en ligne le 01 février 2008, consulté le 25 février 2023. URL : http://journals.openedition.org/archeopages/854 ; DOI : https://doi.org/

10.4000/archeopages.854 
de la théorie moderne de la réception du passé. Il pressent que les monuments ne sont pas seulement des objets de dilection mais des instruments d'intelligibilité qui doivent être dégagés et analysés dans leur contexte. Il faudra longtemps pour que ses recommandations soient acceptées et deviennent la référence en matière de dégagement d'une ville antique.

Le XVIII ${ }^{\mathrm{e}}$ siècle, dans la suite d'Herculanum et de Pompéi, a fait de l'excavation du sol un genre social, littéraire et scientifique. Grâce à cela, les vieilles antiquités sont devenues, au début du XIX ${ }^{\mathrm{e}}$ siècle, l'archéologie tout court... Pourtant, comme le soulignent Montalembert et Hugo, on n'a jamais autant détruit de monuments anciens que durant les premières décennies du $\mathrm{XIX}^{\mathrm{e}}$ siècle (sur tout cela, il existe une large bibliographie; voir Debray 1999). D'où vient que cet équilibre et cette sensibilité, défendus à la tribune du Sénat par Montalembert - «La mémoire du passé ne devient importune que lorsque la conscience du présent est honteuse»-, ne semble plus d'actualité? C'est que les dernières conséquences de la révolution industrielle ont vu surgir les engins mécaniques de déblaiement et que, depuis les années 1930, les travaux d'aménagement détruisent à une vitesse jusqu'alors inconnue des pans entiers du passé sans qu'aucune observation ne soit possible (Schnapp 1998-1999; Vadelorge, Poirrier 2003).

L'archéologie préventive est née de ces destructions. Dans les pays qui ont entrepris, depuis le $\mathrm{XVI}^{\mathrm{e}}$ siècle, de protéger leur patrimoine archéologique tout autant que leur patrimoine écrit, elle ne se distingue guère de l'archéologie tout court car elle a été intégrée dans les procédures de planification et de protection. La Scandinavie a su, avant l'Europe moyenne et méditerranéenne, trouver des compromis acceptables pour sauvegarder les sites archéologiques et documenter ceux qui devaient être détruits. L'Allemagne, l'Italie et l'Angleterre sont venues plus tard. Les deux premiers pays ont entrepris, dès les premières décennies du XIX ${ }^{\mathrm{e}}$ siècle, d'organiser la surveillance de leur patrimoine archéologique et créé des structures efficaces de fouille et d'étude. La Grande-Bretagne n'a pris que tardivement conscience de cet enjeu. Mais le mouvement Rescue, lancé dans les années 1960 par le Council of British Archaeology (une organisation issue de la société civile), a su créer un climat propice au «sauvetage» des sites les plus exposés. En France, nous avons fait lentement et difficilement, depuis les années 1970, l'expérience de la nécessité d'une protection du patrimoine archéologique menacé par les grands travaux. Le pays ne s'est doté d'un embryon de législation archéologique que sous le régime de Vichy, en 1941; il n'a pris conscience de l'ampleur des destructions qu'à compter de 1975 avec le rapport Soustelle. Il est ensuite passé d'une archéologie passive (qui se contentait de laisser faire les destructions)

à une archéologie réactive (qui tentait de les maîtriser). Ce fut la période 1975-2001 et le développement de l'Afan.

Depuis la loi sur l'archéologie préventive de 2001, une troisième période s'est ouverte; elle aussi difficile et complexe mais qui rend possible, en amont des destructions, la prise en compte de leur impact. Les archéologues savent affronter avec des outils adaptés les dangers qui menacent des milliers d'hectares jusqu'alors épargnés. Mais ils ne peuvent convaincre leurs concitoyens de l'intérêt de leurs travaux et des succès qu'ils remportent pour l'histoire qu'en publiant les résultats de leurs recherches. L'archéologie préventive ne tient sa légitimité que d'un dialogue avec le public, une connivence seule à même de créer les conditions d'un consensus pour rendre possible, pendant le cours laps de temps d'un grand chantier, l'observation scientifique. Il ne s'agit donc pas de tout fouiller et encore moins de tout conserver, mais de préserver un équilibre entre la mémoire et l'oubli. Les éradicateurs potentiels ne sont plus ceux qui détruisent les monuments ou les sites anciens, mais ceux qui considèrent qu'un léger prélèvement opéré sur les dépenses d'aménagement est un frein à l'économie ou à l'équilibre des finances publiques. Comme la Grèce ou l'Italie, la France a une histoire faite de monuments et de sites. On ne brûle plus les livres... Préservons les sites. Nous avons sûrement encore besoin des voix de Montalembert et de Hugo.

Borges J. L. 1993: «La muraille et les livres», in Autres inquisitions, repris dans Euvres complètes, t.I, Paris, Gallimard, p.673-675.

Debray R. (dir.) 1999: «L'abus monumental», in Entretiens du Patrimoine, Paris.

Hoffmann V. et al. (dir.) 2005: Die «Denkmalpflege» vor der Denkmalpflege, Berne, Peter Lang.

KAFKA F. 1994: Beim Bau der chinesichen Mauer und andere Schriften aus dem Nachlass, Francfort.

MAFFeI S. 1748: Tre Lettere del del Signor Marchese Scipione Maffei, Vérone, p.33-36.

Nemoy L. 1939: «The Treatise on the Egyptian Pyramids», ISIS, 30, 1, p.17-37.

SCHNAPP A. 1998-1999: «La mémoire qui flanche ou l'impossible normalisation de l'archéologie en France», Le Débat, p.119-132

VAdelorge L., Poirrier P. 2003: Pour une histoire des politiques du patrimoine, Paris, Comité d'histoire du ministère de la Culture, Fondation Maison des sciences de l'homme.

\section{Évolution et professionnalisation de l'archéologie préventive en milieu rural dans le nord de la France}

Marc Talon

Inrap

E Picardie et dans le Nord-Pas-de-Calais, l'archéologie rurale concerne aujourd'hui 80 pour cent des opérations effectuées, se répartissant en deux tiers de sondages et un tiers de fouilles. Vingt années de pratique de l'archéologie préventive en Picardie et dans 


\section{Ce flutiau* du XIVème prouve deux choses: Un, que nos ancêtres savaient en jouer Deux, que la SNCF s'intéresse à nos ancêtres.}

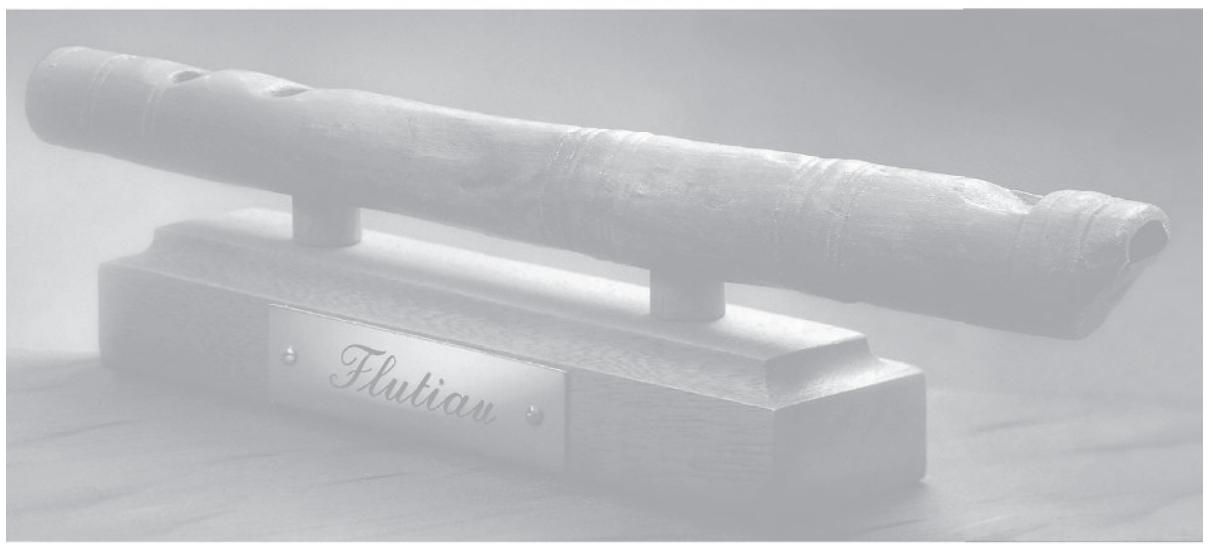

Fouilles archéologiques sur le tracé du TGV Nord Europe.
Un investissement de 60 millions de Francs.
Plus de 100 sites mis à jour par 318 archéologues.
Tous les spécialistes, paléo-environnementalistes, géologues, anthropologues, archéozoologues ou xylologues sont d'accord, les fouilles archéologiques sur le tracé du TGV Nord sont une totale réussite. Cette opération est l'une des plus importantes jamais réalisée sur un tracé linéaire aussi bien au niveau du nombre de chercheurs mobilisés qu'au niveau des moyers mis à leur disposition par la SNCF Sur les $450 \mathrm{kms}$ de tracé, une centaine de sites ont été fouillés. Soit environ un tous les $4 \mathrm{kms}$. Au total, plus de 3 ans ont été nécessaires à cette entreprise, princpalement financée par la SNCF. Un formidable chantier qui a permis de metire à jour de nombreux résors. *Cirdessis, une petite flote aे bec. tournée et complète provenant du site de Serris en Seine-tt-Marne. Une découverte exceptionnelle aux dires de tous les spécialistes.

[Fig. 1] Publicité de la SNCF sur

l'archéologie et le TGv Nord parue en 1990 dans la presse nationale.

Pour la première fois, un aménageur détourne et utilise une découverte archéologique, dans une campagne de presse nationale, dans le but

de valoriser son image. Il s'agit

d'une petite flûte en bois de sureau découverte dans le fossé d'une

maison forte du XIV siècle fouillée sous la direction de B. Foucray (SRA Ile-de-France) et F. Gentili (Inrap) à Serris (Seine-et-Marne). 
le Nord-Pas-de-Calais permettent de présenter un bilan de l'évolution de l'archéologie rurale dans le nord de la France. ${ }^{1}$

Si l'archéologie préventive en milieu urbain a pu émerger et s'organiser dès le début des années 1980, grâce aux travaux effectués par les services de l'État et des collectivités territoriales, il a fallu attendre la fin de ces mêmes années pour constater une véritable professionnalisation en archéologie rurale, qui était jusqu'alors pratiquée soit lors de fouilles programmées accueillant étudiants et bénévoles, soit au cours de sauvetages ouverts en urgence, à l'occasion de découvertes fortuites. Cette professionnalisation s'est faite au gré des occasions et des partenariats noués entre archéologues et aménageurs. Ce sont ces étapes qui sont présentées ici, en privilégiant les phases de terrain, les relations avec les aménageurs et les apports méthodologiques et techniques.

La première opportunité structurante pour le développement de l'archéologie préventive en milieu rural a été l'intervention en carrières. Celles-ci présentaient, à l'époque, un double intérêt: une grande accessibilité et une facilité de repérage des structures archéologiques, en raison de la nécessité, pour le carrier, de décaper la terre végétale sur de grandes surfaces, avant de commencer l'exploitation du sable et des graviers.

Dès les années 1970, un programme scientifique associant le CNRs et l'université ParisI avait été organisé dans la vallée de l'Aisne, justifié par des carrières ouvertes dans les fonds de vallée, zones privilégiées pour les implantations humaines. La mise en place d'un premier partenariat entre exploitants et archéologues a conduit les premiers à décaper leurs sites sous le contrôle des seconds, charge à ces derniers de fouiller bénévolement les vestiges mis au jour. Cependant, devant l'ampleur des destructions qui mitaient peu à peu le paysage et au vu des résultats d'un tel programme, qui permettait à la fois le décapage extensif de grandes surfaces et le recollement progressif de ces décapages, ce partenariat a débouché sur des conventions et sur la mise en place des premiers emplois salariés, grâce aux financements de l'État et des carriers. Dans un troisième temps, l'augmentation des surfaces explorées et l'élargissement des programmes des vallées de l'Aisne et de l'Oise ont débouché sur une convention régionale annuelle, associant l'ensemble des exploitants et liant les différents partenaires par un planning d'intervention. Dès le début des années 1990, on trouve ainsi dans ces conventions une véritable mutualisation avec une redevance à l'hectare et une planification des travaux, un comité de pilotage assurant le suivi de l'ensemble.

Scientifiquement, ces programmes qui portèrent chaque année sur une centaine d'hectares permirent le traitement de très grandes superficies et l'élaboration de méthodes liées à l'étude spatiale des nombreux sites mis au jour. La lecture aisée des structures sur le sable facilite le travail, et la présence, en fond de vallée, de la nappe phréatique favorise la conservation de vestiges en bois et de macrorestes végétaux propices aux études paléoenvironnementales.

La deuxième opportunité a été la construction de la ligne du TGV Nord, tracé linéaire de 45okilomètres reliant les régions Nord-Pas-deCalais, Picardie et Île-de-France, entièrement financée par la SNCF entre 1988 et 1991. Si l'Afan a géré l'ensemble du projet et fourni l'essentiel des intervenants, des archéologues de l'État, des collectivités, du CNRS et de l'Université ont pris en charge une partie des directions de chantier, ce qui a permis de réaliser dans les temps impartis cette opération d'envergure.

Méthodologiquement, elle a consisté à effectuer des sondages sur les secteurs ayant révélé des indices de site lors des études préalables (prospections pédestres et études documentaires. Lorsqu'ils étaient positifs, ces sondages ont été suivis de fouilles effectuées soit à partir de simples fenêtres, soit sous la forme de vastes décapages. Ces derniers se sont limités à l'emprise du projet, hormis deux cas qui ont fait l'objet de fouilles complémentaires: un important site gaulois à Montmartin a ainsi pu être traité sur un «délaissé» entre le tracé du TGV et celui de l'autoroute A1; l'extension d'une nécropole mérovingienne à Baron, dans le champ d'un agriculteur, a fait l'objet d'une fouille programmée. Bien que rares, de tels cas méritent d'être évoqués puisqu'ils permettent de donner toute sa valeur scientifique, patrimoniale et pédagogique à un site comme celui de la villa de Béhen, explorée sur le tracé de l'A28 dans la Somme. Le Tgv Nord a permis de tester d'autres techniques de fouille peu utilisées sous nos latitudes, comme l'archéologie subaquatique en collaboration avec le CNRAs (Centre national de recherches archéologiques subaquatiques) et de réaliser des transects géomorphologiques dans les vallées traversées. Ce travail, effectué avec un carottier et couplé avec les prélèvements réalisés par une équipe permanente de spécialistes, a donné lieu à une vaste étude paléoenvironnementale et suscité des collaborations pluridisciplinaires durables.

En raison d'une enveloppe financière fermée, ce projet a été suivi chaque semaine dans le cadre de réunions entre la SNCF et la coordination État-Afan, l'aménageur ayant un actif rôle de contrôle et de gestion directe des moyens et des équipements. Pour la SNCF, c'était la première expérience de suivi exhaustif d'un tracé - expérience dont elle s'est servi pour sa communication, comme en témoigne une publicité pleine page parue dans de nombreux magazines à l'époque [Fig.1].

La troisième opportunité, devenue pérenne, est celle des diagnostics et des fouilles dans les zones d'aménagement concerté ( $\mathrm{Zac})$. Au début des années 1990, influencés par les pratiques des archéologues lorrains, les premiers diagnostics systématiques par tranchées de sondages couvrant 1opour cent de la surface d'emprise de la Zac étudiée ont été lancés. Les secteurs positifs faisaient l'objet de décapages et de fouilles, 

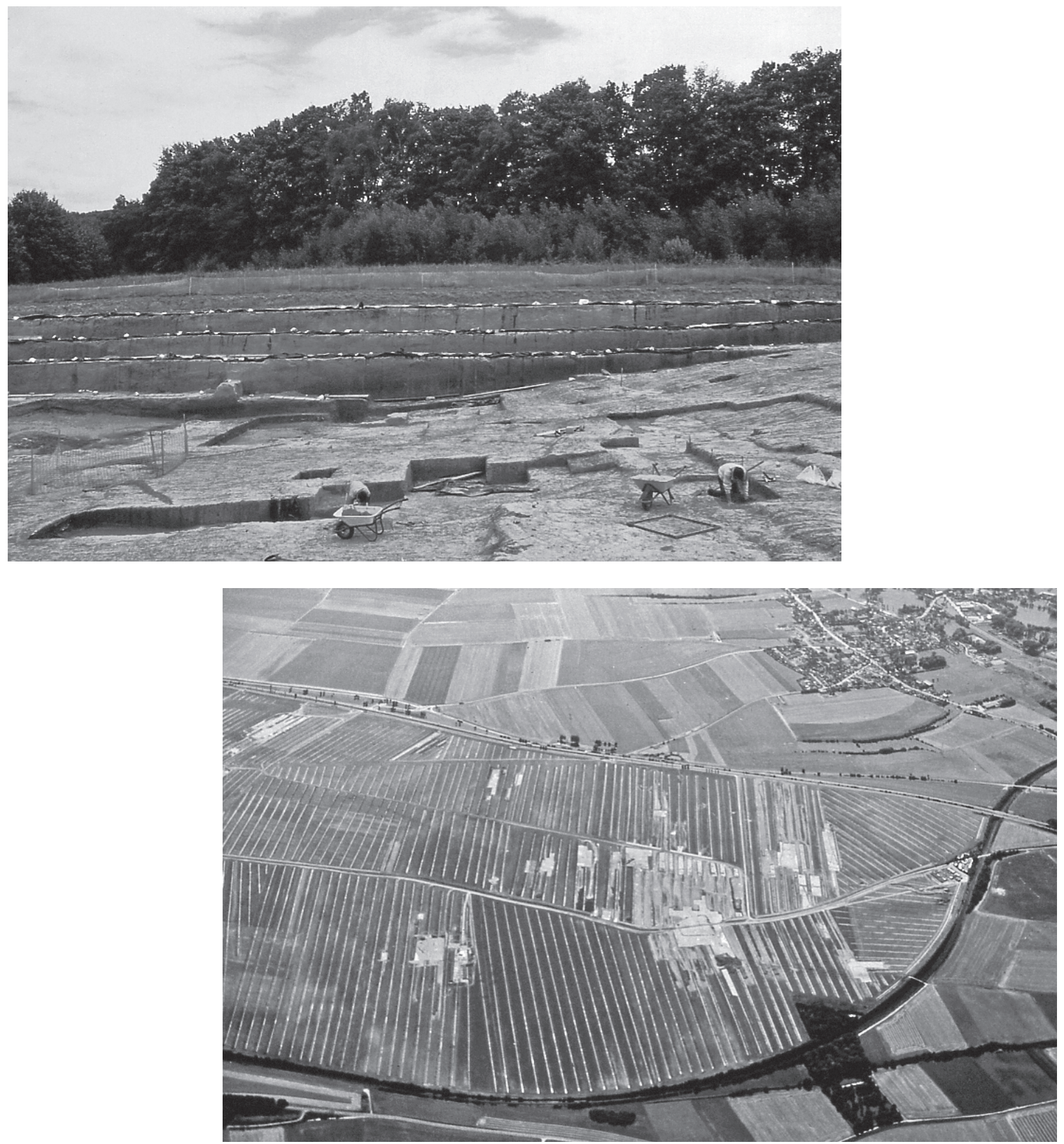

[Fig.2] Décaissement en gradins pour l'étude sécurisée du site du Paléolithique moyen de Fresnoyle-Luat (Somme). La mise en œuvre de moyens de terrassement importants permet, sur de grandes surfaces, l'étude de gisements de plein air de la Préhistoire ancienne. Ici la fouille conduite par J.-L. Locht (Inrap)

sur ce site découvert sur le tracé de l'autoroute A29 a notamment mis en évidence une occupation datée de plus de 100000 ans et identifiée dans la stratigraphie par un sol gris-forestier (au premier plan).
[Fig.3] Vue aérienne du site Actiparc près d'Arras (Pas-de-Calais). Ce vaste programme réalisé en une vingtaine de mois et qui portait sur plus de 300 hectares, dont 63 ont été fouillés, a été l'occasion d'une codirection entre l'Inrap

(G. Prilaux) et le service

archéologique de la ville d'Arras (A. Jacques). Il a notamment permis la mise au jour d'un vaste domaine aristocratique atrébate auquel succède un poste militaire romain. Ces travaux complètent de façon

spectaculaire l'étude de la ville de Nemetacum, la future Arras, et l'histoire de la romanisation en Gaule. 
financés par le truchement de conventions négociées individuellement avec les aménageurs. Les partenaires étaient cette fois-ci des collectivités: commune, syndicat intercommunal ou département. Ces opérations ont permis d'explorer tous les types de substrat (puisqu'elles n'étaient pas limitées aux vallées ou à des tracés linéaires), de documenter les plateaux et d'autres points des fonds de vallée délaissés par les carriers mais occupés par des chenaux anciens et des berges fossiles de rivière. Une nouvelle méthode a été élaborée avec des géomorphologues et des paléoenvironnementalistes, pour cartographier la dynamique alluviale. Elle a favorisé la mise au jour de paléochenaux stratifiés, contenant des niveaux organiques propices au piégeage de vestiges en bois conservés et des îlots où des habitats avaient été scellés sous des limons de débordement.

Parallèlement à la mise en place de ces diagnostics, une nouvelle expérience d'archéologie préventive liée à la construction de l'autoroute A16 s'est organisée. Ici, la collaboration entre l'Afan, le service régional de l'archéologie de Picardie et l'aménageur - la Sanef - a pris une tournure décisive, les archéologues étant considérés comme de véritables professionnels avec lesquels étaient définis le planning des opérations et l'utilisation des moyens de terrassement. Les diagnostics, devenus systématiques, ont couvert l'ensemble du tracé. Restait quelques différends qui portaient sur le débat sur les déblais et les remblais, questions qui ont resurgi avec la notion «d'éléments du patrimoine archéologique affectés ou susceptibles d'être affectés», figurant dans l'article premier de la loi du 17janvier 2001. À cette époque, les archéologues - inspirés par des pratiques mises au point sur l'A 5 et le TGV Nord - effectuaient des sondages profonds systématiques permettant l'exploration des loss; accumulations sédimentaires récentes typiques du Bassin parisien, ces loss contenaient des niveaux préhistoriques qui neétaient jusqu'alors repérés qu'à l'occasion des grands terrassements effectués lors des aménagements.

Le développement de ces sondages, mis en œuvre en respectant la côte de fond de forme afin d'éviter toute déstabilisation du substrat, a été accompagné, dans un souci sécuritaire, par l'élaboration de cages de sécurité. Reste que le décaissement sur des emprises plus larges est le moyen le plus efficace pour étudier sereinement ces occupations paléolithiques [Fig.2].

Très développé, le partenariat avec la Sanef s'est construit sur près de dix ans de collaboration à travers quatre opérations. Il a coïncidé avec la structuration de l'Afan au moment de la création des antennes, la mise en place des coûts d'unité d'œuvre et de la politique d'hygiène et de sécurité. Les relations avec cet aménageur ont été particulièrement constructives dans cette professionnalisation.

Plus récente, la dernière étape est particulière au nord de la France. Elle est illustrée par de très grands projets pour lesquels les aménageurs (chambres de commerce et d'industrie, communautés de communes) sont pressés de voir intervenir les archéologues... et libérer les terrains. Le cas le plus emblématique a été le projet Toyota, concernant 23ohectares à étudier en six mois, l'Afan devant assumer complètement les marchés de terrassements, la base vie, l'arrivée en quinze jours et en plein hiver de cinquante archéologues et d'une dizaine de pelles mécaniques. Depuis, d'autres projets sont venus compléter cette expérience, tels Dourges et Villeneuve-d'Ascq avec chacun 140 hectares, Arras-Actiparc avec 310 hectares [Fig.3] ou Albert-Méaulte avec 120hectares. Là, ce sont les notions de délai et de prise en charge de l'ensemble de l'intervention, terrassements compris, qui ont primé.

Ces vastes opérations montrent ce que l'archéologie préventive est à même de faire dans les conditions les plus extrêmes, lorsque les moyens lui en sont donnés. Sur des territoires qui ne sont pas toujours bien documentés, elle a inventé et mis en application des méthodes adaptées au terrain et aux contraintes de calendrier, en collaboration étroite avec les aménageurs. Enfin, elle a recueilli, sur des substrats et dans des régions différentes, des données importantes qui sont venues enrichir les modèles d'occupation du paysage proposés à partir des programmes sablières, premières opportunités structurantes de notre démonstration.

BLANCQUAERT G. 2006: «Étude comparative de 4opération archéologiques réalisées en milieu rural dans la région Nord-Pas-de-Calais - France», in G. Blancquaert et M. Medlycott (dir.), Archaeological Evaluation of Rural Areas in the Planarch Area of North West Europe, Planarch 2: Action 2C, Maidstone, p.19-38, 16fig.

BRUn P., Demoule J.-P. 200o: «Vingt-cinq ans de sauvetage archéologique dans la vallée de l'Aisne», in «Recherche et Archéologie préventive», CNRS Info, hors série, p.16-17.

Brun P., Marcigny C., VANMOerkerke J. (dir.) 2006: "Une archéologie des réseaux locaux. Quelles surfaces étudier pour quelle représentativité?», Actes de la table ronde des 14 et 15 juin 2005 à Châlons-en-Champagne, Les Nouvelles de l'archéologie, $\mathrm{n}^{\circ} 104$.

Catteddu I., Roger D. 2002: «L'opération Onnaing-Toyota en 2 parties», Archéologia, n ${ }^{\circ} 385$, janvier, p.48-57, et n ${ }^{\circ} 386$, février 2002, p. 42-51.

Collectif 1991: Archéologie de la vallée de l'Oise, Compiègne et sa région depuis les origines, catalogue d'exposition, CRAvo, Compiègne.

Collectif 1991: Archéologie d'une vallée: la vallée de l'Aisne des derniers chasseurs-cueilleurs au premier royaume de France, catalogue de l'exposition «Trente ans d'archéologie dans la vallée de l'Aisne», Soissons, ADMs.

Collectif 1994: Archéologie et aménageurs, Lyon, Centre d'étude sur les réseaux, les transports, l'urbanisme et les constructions publiques.

COLLECTIF 2005: «La Recherche archéologique en Picardie: bilans et perspectives», Revue archéologique de Picardie, $\mathrm{n}^{\circ}$ 3-4.

JaCQues A., Prilaux G. 2003: Dans le sillage de César: traces de romanisation d'un territoire, les fouilles d'Actiparc à Arras catalogue d'exposition présentée au Musée des Beaux-Arts d'Arras.

LOMBARDO J.-L. 1994: «L'accessibilité aux terrains: l'exemple de l'autorouteA16», in «L'Archéologie préventive en milieu rural et des phases d'évaluation», Actes de la table ronde de Dijon 1993, Nouvelles de l'archéologie, $\mathrm{n}^{\circ} 58$, p.11-15.

Saint-Blanquat H. De 1992: Archéo TGV, 45okm d'histoire, Paris, Casterman.

TALON M. 1994: «Prospection et évaluation sur le tracé du TGV Nord et de l'interconnexion», in "L'Archéologie préventive en milieu rural et des phases d'évaluation», Actes de la table ronde de Dijon 1993, Nouvelles de l'archéologie, ${ }^{\circ} 58$, p. 25-29.

TALON M. 1999: «La pratique de l'archéologie environnementale à l'Afan», in «Environnement et archéologie», Actes de la table ronde de Nanterre 1999, Nouvelles de l'archéologie, nº78, p.29-31. 